\title{
Household number associated with middle ear disease at an urban Indigenous health service: a cross-sectional study
}

\author{
Geoffrey K. P. Spurling A,B,G, Deborah A. Askew ${ }^{\mathrm{A}, \mathrm{B}}$, Philip J. Schluter ${ }^{\mathrm{C}, \mathrm{D}}$, Fiona Simpson ${ }^{\mathrm{E}}$ \\ and Noel E. Hayman ${ }^{\mathrm{B}, \mathrm{F}}$ \\ ADiscipline of General Practice, The University of Queensland, Royal Brisbane and Women's Hospital, \\ Herston, Qld 4029, Australia. \\ ${ }^{B}$ Inala Indigenous Health Service, Queensland Health, 64 Wirraway Parade, Inala, QId 4077, Australia. \\ ${ }^{\mathrm{C}}$ School of Health Sciences, University of Canterbury, Christchurch 8140, New Zealand. \\ DSchool of Nursing and Midwifery, The University of Queensland, Brisbane, Qld 4072, Australia. \\ EPrincess Alexandra Hospital, Queensland Health, Brisbane, Qld 4102, Australia. \\ FSchool of Medicine, The University of Queensland, Herston, Qld 4029, Australia. \\ ${ }^{\mathrm{G}}$ Correspondending author. Email: g.spurling@uq.edu.au
}

\begin{abstract}
Few epidemiological studies of middle ear disease have been conducted in Aboriginal and Torres Strait Islander populations, yet the disease is common and causes hearing impairment and poorer educational outcomes. The objective of this study is to identify factors associated with abnormal middle ear appearance, a proxy for middle ear disease. Aboriginal and Torres Strait Islander children aged 0-14 years receiving a Child Health Check (CHC) at an urban Indigenous Health Service, Brisbane, Australia were recruited from 2007 to 2010. Mixed-effects models were used to explore associations of 10 recognised risk factors with abnormal middle ear appearance at the time of the $\mathrm{CHC}$. Ethical approval and community support for the project were obtained. Four hundred and fifty-three children were included and 54\% were male. Participants were Aboriginal (92\%), Torres Strait Islander (2\%) or both (6\%). Abnormal middle ear appearance was observed in $26(6 \%)$ children and was significantly associated with previous ear infection (odds ratio (OR), 8.8; 95\% confidence interval (CI), 3.2-24.0) and households with eight or more people (OR, 3.8; 95\% CI, 1.1-14.1) in the imputed multivariable mixed-effects model. No significant associations were found for the other recognised risk factors investigated. Overcrowding should continue to be a core focus for communities and policy makers in reducing middle ear disease and its consequences in Aboriginal and Torres Strait Islander peoples.
\end{abstract}

Received 23 August 2012, accepted 7 May 2013, published online 28 May 2013

\section{Introduction}

Middle ear disease is the most common cause of acquired hearing loss in children (Roberts et al. 2004). Rates of middle ear disease, which includes otitis media, vary among different populations, with Australian Aboriginal children having rates among the highest in the world (Couzos et al. 2001).

Large birth cohort studies in the Netherlands and the United States of American (USA) found that significant predictors of otitis media at 2 years of age were male gender, otitis media in the first year of life, socioeconomic disadvantage and exposure to other children in the house (Paradise et al. 1997; Labout et al. 2011). Higher rates of otitis media among Aboriginal and Torres Strait Islander children have been attributed to early nasopharyngeal bacterial colonisation, chronic upper respiratory tract infections and social factors such as poverty, overcrowding and exposure to cigarette smoke (Kong and Coates 2009). However, surprisingly few epidemiological studies have been conducted to investigate the risk factors for middle ear disease in this population, and those that do yielded varied findings (Jacoby et al. 2008; Bailie et al. 2012). Most of this research has been confined to populations residing in rural or remote communities, yet Australian Aboriginal and Torres Strait Islander peoples now predominantly live in urban areas, with $53 \%$ living in major cities or regional centres (Australian Bureau of Statistics 2007).

The objective of this study is to detect factors associated with abnormal middle ear appearance, a proxy for middle ear disease, in children attending an urban Indigenous health service.

\section{Methods \\ Setting}

The Inala Indigenous Health Service (IIHS) is a Queensland Government primary health care facility, located in south-western Brisbane, Australia, and predominantly cares for Aboriginal and Torres Strait Islander people from surrounding suburbs. This study recruited a consecutive sample of Aboriginal and Torres Strait Islander children, aged 0 to 14 years, receiving a Child Health Check (CHC) at the IIHS from March 2007 to March 2010. 


\section{What is known about the topic?}

- Middle ear disease is common with particularly high rates among Aboriginal and Torres Strait Islander people. The few epidemiological studies that have been conducted have been in rural/remote contexts.

\section{What does this paper add?}

- This paper finds an association between increasing household number and abnormal middle ear appearance for Aboriginal and Torres Strait Islander children attending an urban primary health care service.

Parents or carers consented for their child's de-identified CHC data to be included in this study. For children who had more than one $\mathrm{CHC}$ during the study period, only data from the first $\mathrm{CHC}$ were included in the analysis.

\section{Outcome variable}

We defined abnormal appearance of the middle ear as any abnormal appearance of the middle ear at the time of the $\mathrm{CHC}$, specifically the presence or absence of bulging ear drums, wet perforation or dry perforation. This definition is clinically relevant and was consistent with criteria used by Indigenous CHCs in remote contexts (Spurling et al. 2012). It is likely to include children with otitis media on the day of the health check, children with otitis media with effusion and children with residual middle ear problems such as perforated tympanic membranes from previous infections. Otitis media, as opposed to middle ear disease, is a difficult diagnosis to standardise, especially as GPs are reluctant to employ pneumatic otoscopy or tympanometry even after appropriate training (Rosenkranz et al. 2012).

\section{Independent variables}

Ten recognised risk factors for middle ear disease were included in the CHC: four host factors (age, gender, preterm gestation ( $<37$ weeks) and parent-reported previous ear infection) and six environmental factors (exposure to household tobacco smoke (parent report), immunisations not up to date (as per the child's medical record), never breastfed (parent report), regular attendance at childcare or school at the time of the health check (parent report), presentation for a $\mathrm{CHC}$ in a winter month (based on the date of the health check), and the number of people living in the house at the time of the health check (parent report)).

For this last variable, we assumed a priori that households with eight people or more were likely to meet the definition of overcrowding given the rarity of houses with more than four bedrooms in Inala (Hayman et al. 2002). Higher household number may also be associated with middle ear disease independent of crowding through exposure to siblings. In recognition of this and the non-standard measure for overcrowding, analyses were also conducted using different household number thresholds from five to nine. Swimming, and other environmental and socioeconomic factors thought to modify the risk of middle ear disease were not available.

\section{Analysis}

Data from participants' CHCs were manually entered into an Excel spreadsheet, then imported into specialist statistical software packages SAS version 9.2 (SAS Institute, Cary, NC, USA) and Stata version 10.0 (StataCorp, College Station, TX, USA) for analysis and graphing, respectively. Categorical outcomes are presented as percentages and continuous outcomes as medians and first $\left(\mathrm{Q}_{1}\right)$ and third quartiles $\left(\mathrm{Q}_{3}\right)$. The gamma statistic, which measures the surplus of concordant pairs over discordant pairs as a percentage of all pairs, ignoring ties, and ranges between +1 to -1 , was used to assess the relationship between two binary variables.

Recognising that children are nested within families and that the infectious nature of otitis media may lead to a clustering of children with middle ear disease in households with some dependent correlations, mixed-effects models were used for all analyses to explore the associations of recognised risk factors with abnormal middle ear appearance. In these models children were clustered within families, and families were treated as random effects (using the GLIMMIX procedure with the logistic regression option). Crude analyses were first investigated. Gender, age and the eight other recognised risk factors measured by the $\mathrm{CHC}$ were then simultaneously included into a multivariable model. As some of these covariates contained missing or invalid data, this multivariable analysis was undertaken using imputed data. A multistage multiple imputation method was employed (using the MI procedure), using successive nonparametric propensity score and logistic regression methods to impute values for continuous and binary variables, respectively. For analyses involving imputed data, $\mathrm{m}=50$ datasets were generated, analysed, and results reported for various household number thresholds (using the MIANALYSE procedure). A significance level of $5 \%$ was used for all statistical tests.

\section{Ethics}

Ethics approval for this study was obtained from the University of Queensland's Behavioural and Social Science Ethics Research Committee and the Metro South Human Research Ethics Committee. The Inala Elders Aboriginal and Torres Strait Islander Corporation supported the project.

\section{Results}

There were $453 \mathrm{CHCs}$ available for analysis. The median age was 6 years $\left(\mathrm{Q}_{1}=2, \mathrm{Q}_{3}=9\right.$ years), $222(49 \%)$ children were aged between 0 and 5 years, and 207 (46\%) were female. Participants were Aboriginal (92\%), Torres Strait Islander (2\%) or both Aboriginal and Torres Strait Islander (6\%).

Abnormal middle ear appearance was observed in $26(6 \%)$ children and was mostly owing to a bulging tympanic membrane (Table 1). Table 1 also presents the prevalence of recognised risk factors for middle ear disease. Fifty-four percent (246) of the children were male, $114(25 \%)$ had a previous ear infection and $45(10 \%)$ reported preterm birth (less than 37 weeks gestation). Childcare attendance and exposure to environmental tobacco smoke were both present for around $70 \%$ of children in this study. The median household number reported was five $\left(\mathrm{Q}_{1}=4, \mathrm{Q}_{3}=6\right)$, with a maximum of 12 , and $42(9 \%)$ children 
Table 1. Outcomes for middle ear disease and recognised risk factors from Child Health Checks at the Inala Indigenous Health Service $(n=453)$

\begin{tabular}{lr}
\hline Middle ear appearance and risk factors & $n(\%)$ \\
\hline Dependent variable & \\
Abnormal otoscopic middle ear appearance (one ear or both) & $26(6)$ \\
Bulging tympanic membrane (one ear or both) & $17(4)$ \\
Dry perforation (one ear or both) & $5(1)$ \\
Wet perforation (one ear or both) & $5(1)$ \\
Independent variables & \\
Host risk factors & \\
Age, median years (quartile 1, 3) & $6(2,9)$ \\
Gender (male) & $246(54)$ \\
Preterm (born <37 weeks gestation) & $45(10)$ \\
Environmental risk factors & \\
Attendance at day care/school & $296(77)$ \\
Household smoking exposure & $332(73)$ \\
Never breastfed & $117(33)$ \\
Immunisation due & $145(32)$ \\
Previous ear infection & $114(25)$ \\
Presenting for CHC in a winter month & $77(17)$ \\
Eight or more people living in household & $42(9)$ \\
\hline
\end{tabular}

lived in households with eight or more usual residents (Table 1).

The frequencies of abnormal middle ear presentation, crude odds ratios (OR) and associated 95\% confidence intervals (CI) estimated from mixed-effects models are presented in Table 2. Perusal of this table reveals that previous ear infection was the only investigated risk factor significantly associated with abnormal middle ear appearance (OR, 5.2; 95\% CI, 2.3-11.9). However, a household number of eight or more (OR, 1.8; 95\% CI, 0.6-5.7) and presenting in the winter months (OR, 1.9; 95\% CI, $0.8-4.7$ ) both have moderate but non-significant effect sizes with OR approaching 2. Complete data on both the dependent and independent variables were only available for $253(56 \%)$ children, with gestational data missing for 124 (27\%), breastfeeding data missing for 95 (21\%), childcare information absent for 70 (15\%), household size data missing for $23(5 \%)$, and previous ear infection information missing for eight $(2 \%)$ children. Imputing the missing data, then undertaking multivariable mixed-effects model analysis yielded the adjusted OR and associated $95 \%$ CI also contained in Table 2. Significant associations with abnormal middle ear appearance in these imputed analyses was seen for a history of ear infections (OR, 8.8; 95\% CI, 3.2-24.0) and a household number of eight or more people (OR, 3.8; 95\% CI, 1.1-14.1). Results from multivariable logistic regression analyses where the ratio of the number of outcome events (abnormal middle ear appearance) to the number of independent variables is low and may result in regression coefficients which are biased in both positive and negative directions (Peduzzi et al. 1996). Similar results were obtained when only the three variables with the largest effect size (previous ear infection, household number of eight or more and presenting in a winter month) were included in the multivariable mixedeffects model analysis (Table 2). The estimated gamma statistic measuring the relationship between previous ear infection and household number (eight or more) was 0.67 (asymptotic standard error 0.17 ), which suggests that this relationship has led to a higher adjusted OR for abnormal middle ear appearance than the crude OR. When children are not indicated as having a previous ear infection, then the imputed adjusted OR for abnormal middle ear associated with household number (eight or more) was 5.9 (95\% CI, 1.5-23.2; $P=0.01)$. The imputed adjusted OR for abnormal middle ear disease was not calculable for those indicated as having previous ear infection. The adjusted OR for abnormal middle ear appearance increased with increasing household number thresholds (Fig. 1). Moderate but nonsignificant effect sizes were seen for presenting in the winter months (OR, 2.5; 95\% CI, 0.9-6.7) and immunisation history (OR, 2.0; 95\% CI, 0.8-5.0). Other risk factors measured in this study were not associated with abnormal middle ear appearance. There were no significant differences for any outcomes when the populations were divided into two age groups ( $0-4$ years, 5-14 years), although the associated statistical power was relatively small.

\section{Discussion}

Middle ear disease in this study was associated with previous ear infection and increasing household number in a dose-dependent manner for children attending the urban IIHS for their CHC. Middle ear disease was less prevalent in this urban context than in previous studies in rural/remote Aboriginal communities (Morris et al. 2005; Bailie et al. 2010).

This study has several strengths and limitations. Clinical assessment of middle ear disease, while not standardised, was conducted by primary GPs with appropriate training to examine ears, and a degree of accuracy might reasonably be assumed. The broad nature of the $\mathrm{CHC}$ allowed for the collection of data relevant to many of the recognised factors for middle ear disease in children. A limitation of this study was the use of a consecutive sample of children attending a primary health care service. While the gender distribution in this study was similar to corresponding 2006 Census data from Inala (54\% males compared with 52\% in Census data) (Australian Bureau of Statistics 2006), 6-14-yearolds were under-represented (51\% compared with $70 \%$ of $0-14$ year-olds in Census data). The apparent under-representation of school-age children in this study may be due to decreased frequency of attendance for primary health care because of being at school, or when they do attend, they may be too sick to have a CHC.

Some information regarding risk factors for middle ear disease were missing, requiring utilisation of multiple imputation methods. The relationship between previous ear infection and household number (eight or more) may be one of effect modification rather than confounding; however, the sample size was too small to make any definitive statements. Information regarding risk factors that was collected may be subject to parental recall bias. Data collected in this study represent one urban context and might not be generalisable to other urban Indigenous health services, although there is little reason to assume that there would be substantial differences in the results. While the $\mathrm{CHC}$ did not collect sufficient information, such as number of bedrooms, to use recognised measures of overcrowding (Schluter et al. 2007), we found that $9 \%$ of participants lived in households with eight or more people. In a recent national survey, overcrowding was 
Table 2. Crude and adjusted odds ratios (OR) with associated $95 \%$ confidence intervals (CI) of abnormal middle ear appearance during Child Health Checks at the Inala Indigenous Health Service

\begin{tabular}{|c|c|c|c|c|c|c|c|c|}
\hline & \multicolumn{2}{|c|}{ Abnormal middle ear } & \multicolumn{2}{|r|}{ Crude } & \multicolumn{2}{|c|}{ Adjusted $^{\mathrm{A}}$ (imputed) } & \multicolumn{2}{|c|}{ Adjusted $^{\mathrm{B}}$ (imputed) } \\
\hline & $\mathrm{N}$ & $n(\%)$ & OR & $(95 \% \mathrm{CI})$ & OR & $(95 \% \mathrm{CI})$ & OR & $(95 \% \mathrm{CI})$ \\
\hline \multicolumn{9}{|c|}{ Previous ear infection } \\
\hline Yes & 114 & $16(14)$ & 5.2 & $(2.3-11.9)$ & 8.8 & $(3.2-24.0)$ & 6.9 & $(2.8-7.0)$ \\
\hline No & 331 & $10(3)$ & 1 & (reference) & & & & \\
\hline \multicolumn{9}{|c|}{ Household number (eight or more) } \\
\hline Yes & 42 & $4(10)$ & 1.8 & $(0.6-5.7)$ & 3.8 & $(1.1-14.1)$ & 3.8 & $(1.0-12.1)$ \\
\hline No & 388 & $21(5)$ & 1 & (reference) & & & & \\
\hline \multicolumn{9}{|c|}{ Presents in winter month } \\
\hline Yes & 77 & $7(9)$ & 1.9 & $(0.8-4.7)$ & 2.5 & $(0.9-6.7)$ & 2.1 & $(0.8-5.5)$ \\
\hline No & 376 & $19(5)$ & 1 & (reference) & & & & \\
\hline \multicolumn{9}{|c|}{ Immunisation not up to date } \\
\hline Yes & 145 & $9(6)$ & 1.1 & $(0.5-2.6)$ & 2 & $(0.8-5.0)$ & & \\
\hline No & 308 & $17(6)$ & 1 & (reference) & & & & \\
\hline \multicolumn{9}{|c|}{ Preterm birth ( $<37$ weeks gestation) } \\
\hline Yes & 45 & $3(7)$ & 1.3 & $(0.4-4.7)$ & 1.6 & $(0.4-6.2)$ & & \\
\hline No & 284 & $15(5)$ & 1 & (reference) & & & & \\
\hline \multicolumn{9}{|l|}{ Never breast fed } \\
\hline Yes & 117 & $8(7)$ & 1.3 & $(0.5-3.2)$ & 1.5 & $(0.6-4.1)$ & & \\
\hline No & 241 & $13(5)$ & 1 & (reference) & & & & \\
\hline \multicolumn{9}{|l|}{ Age (years) } \\
\hline Each year older & 453 & & 1.0 & $(0.9-1.1)$ & 1.0 & $(0.9-1.1)$ & & \\
\hline \multicolumn{9}{|c|}{ Smoker in the household } \\
\hline Yes & 332 & $19(6)$ & 1.0 & $(0.4-2.4)$ & 1.0 & $(0.4-2.6)$ & & \\
\hline No & 121 & $7(6)$ & 1 & (reference) & & & & \\
\hline \multicolumn{9}{|c|}{ Child attended day care/school } \\
\hline Yes & 296 & $18(6)$ & 1.3 & $(0.4-4.1)$ & 0.9 & $(0.2-4.2)$ & & \\
\hline No & 87 & $4(5)$ & 1 & (reference) & & & & \\
\hline \multicolumn{9}{|l|}{ Gender } \\
\hline Male & 246 & $12(5)$ & 0.7 & $(0.3-1.6)$ & 0.7 & $(0.3-1.7)$ & & \\
\hline Female & 207 & $14(7)$ & 1 & (reference) & & & & \\
\hline
\end{tabular}

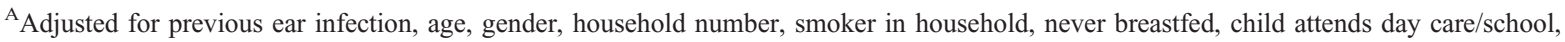
presenting in a winter month, preterm $(<37$ weeks), immunisation not up to date.

${ }^{\mathrm{B}}$ Adjusted for previous ear infection, household number, presenting in a winter month.

experienced by $13 \%$ of Aboriginal and Torres Strait Islander people living in major cities, indicating that our proxy measure may underestimate overcrowding (Australian Institute of Health and Welfare 2008). In the same survey, overcrowding was more likely to be reported in remote communities (3.6 times). Like the higher rate of overcrowding, we have previously reported a higher rate of middle ear disease, detected using $\mathrm{CHCs}$, in remote communities compared with the urban IIHS (5.5 times) (Spurling et al. 2012).

This study's findings regarding the association between middle ear disease and previous episodes of ear infection and increasing household number are consistent with large international cohort studies (Labout et al. 2011), one of which also identified low socioeconomic status as a risk factor (Paradise et al. 1997). However, our findings differ from the two other epidemiological studies involving Aboriginal populations in rural and remote areas (Jacoby et al. 2008; Bailie et al. 2010) and a meta-analysis examining risk factors for middle ear disease (Uhari et al. 1996), which found that environmental tobacco smoke and childcare attendance were important risk factors. In the Kalgoorlie cohort study, overcrowding, defined as more than one person per room, was not significantly associated with specialistdiagnosed otitis media. A follow-up study on the same children found that the best predictor of nasopharyngeal carriage of pathogenic bacteria for otitis media was a more formal evaluation of overcrowding involving the number of people in the house with respect to the number of bedrooms (Jacoby et al. 2011). The Northern Territory study by Bailie et al. (2010) may have underestimated rates of otitis media by relying on carer's recall of an ear infection in the preceding 2 weeks. This would be a potentially difficult diagnosis for the parent of an infant (Engel et al. 2000), and may explain why associations with measures such as crowding were not found (Bailie et al. 2010).

The association of middle ear disease with household number in this study should not be interpreted as causal given the cross-sectional nature of the data. However, in addition to the plausible mechanism of an association between pathogenic nasopharyngeal bacterial carriage with overcrowding and 


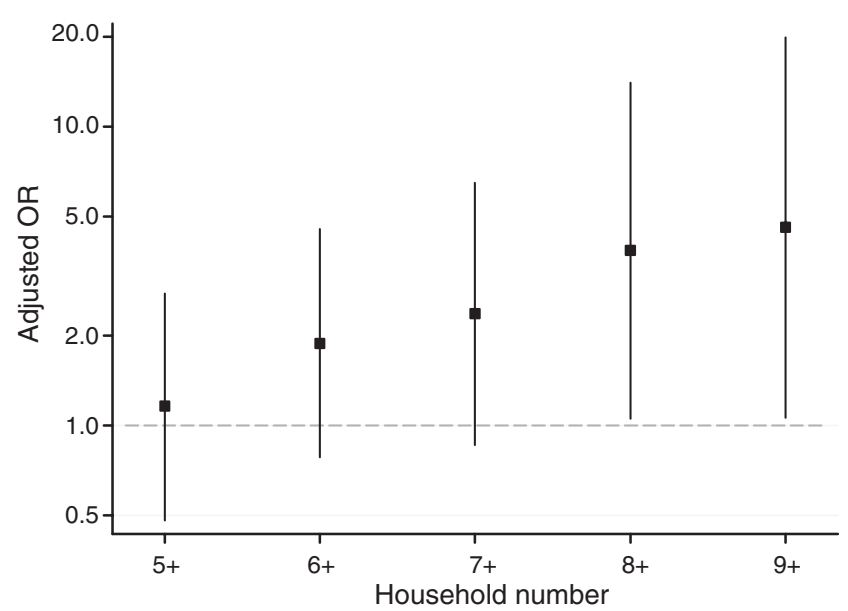

Fig. 1. Household number and middle ear disease at the Inala Indigenous Health Service. Odds ratios (OR) and associated $95 \%$ confidence intervals of household number and middle ear disease at the Inala Indigenous Health Service derived from imputed multivariable mixed-effects models. OR adjusted for previous ear infection, age, gender, smoker in household, never breastfed, child attends daycare/school, presenting in a winter month, preterm ( $<37$ weeks), immunisation not up to date.

specialist-diagnosed otitis media (Jacoby et al. 2011), our finding that increasing household number is associated with increasing prevalence of middle ear disease fulfils the Hill criteria used to establish a causal relationship (Hill 1965). Perhaps surprisingly, the high rates of environmental tobacco smoke exposure and childcare/school attendance found in this study were not associated with middle ear disease. While formal childcare may offset the risk of environmental tobacco smoke, exposure to tobacco smoke has not been shown to predict naso-pharyngeal carriage of pathogenic bacteria after adjusting for confounding factors (Jacoby et al. 2011). Paradise et al. (1997) suggest that environmental tobacco smoke in the context of otitis media, diagnosed using pneumatic otoscopy and tympanometry, is predominantly a proxy for socioeconomic disadvantage (Paradise et al. 1997), a finding not contradicted by this study. Couzos et al.' s systematic review published in 2001 suggested that it was very likely that observations showing associations between middle ear disease and childcare, family size and sibling infection were related to housing factors, poverty and overcrowding (Couzos et al. 2001). Our study, conducted in a low socioeconomic region, supports this insight, especially with respect to household number.

This study may stimulate more questions for researchers regarding the role of risk factors for middle ear disease but the message for policy makers remains clear. Further research may be warranted to clarify how formal childcare may be a protective, neutral or risk factor for middle ear disease. The current policies on hearing health for Indigenous children continue to be solely reactive, addressing needed hearing health services (Australian Government 2012). Indigenous communities and policy makers, working together, need to continue to promote the resolution of socioeconomic issues, such as housing, community conditions and specifically overcrowding in order to reduce the high prevalence of middle ear disease in Aboriginal and Torres Strait Islander peoples.

\section{Conflicts of interest}

None declared.

\section{References}

Australian Bureau of Statistics (2006) 'Indigenous status by age by sex for 4077 postal area, Qld.' Cat. no. 2068.0, 2006 census tables. (Australian Bureau of Statistics: Canberra)

Australian Bureau of Statistics (2007) 'Population distribution, Aboriginal and Torres Strait Islander Australians, 2006.' Cat. no. 4705.0. (Australian Bureau of Statistics: Canberra)

Australian Government (2012) 'Closing the Gap Prime Minister's Report.' (Department of Families Housing Community Services and Indigenous Affairs: Canberra)

Australian Institute of Health and Welfare (2008) 'Housing circumstances: overcrowding.' 4704.0 - The Health and Welfare of Australia's Aboriginal and Torres Strait Islander Peoples. (Australian Bureau of Statistics: Canberra)

Bailie R, Stevens M, McDonald E, Brewster D, Guthridge S (2010) Exploring cross-sectional associations between common childhood illness, housing and social conditions in remote Australian Aboriginal communities. BMC Public Health 10, 147doi:10.1186/1471-2458-10147

Bailie RS, Stevens M, McDonald EL (2012) The impact of housing improvement and socio-environmental factors on common childhood illnesses: a cohort study in Indigenous Australian communities. Journal of Epidemiology and Community Health 66, 821-831. doi:10.1136/ jech.2011.134874

Couzos S, Metcalf S, Murray R (2001) 'Systematic review of existing evidence and primary care guidelines on the management of otitis media in Aboriginal and Torres Strait Islander populations.' (OATSI: Canberra) Available at http://www.health.gov.au/internet/main/publishing.nsf/ Content/health-oatsih-pubs-Syst+review [Verified 13 August 2012]

Engel J, Anteunis L, Volovics A, Hendriks J, Marres E (2000) Predictive value of parent-reported symptoms in the assessment of otitis media with effusion during infancy. Scandinavian Journal of Primary Health Care 18, 25-29. doi:10.1080/02813430050202514

Hayman NE, Kanhutu J, Brady J (2002) 'Urban Aboriginal and Torres Strait Islander Health and Nutrition Survey, South Brisbane, Inala.' (Queensland Health: Brisbane)

Hill AB (1965) The environment and disease: association or causation? Proceedings of the Royal Society of Medicine 58, 295-300.

Jacoby PA, Coates HL, Arumugaswamy A, Elsbury D, Stokes A, Monck R, Finucane JM, Weeks SA, Lehmann D (2008) The effect of passive smoking on the risk of otitis media in Aboriginal and non-Aboriginal children in the Kalgoorlie-Boulder region of Western Australia. The Medical Journal of Australia 188, 599-603.

Jacoby P, Carville KS, Hall G, Riley TV, Bowman J, Leach AJ, Lehmann D (2011) Crowding and other strong predictors of upper respiratory tract carriage of otitis media-related bacteria in Australian Aboriginal and nonAboriginal children. The Pediatric Infectious Disease Journal 30, 480-485.

Kong K, Coates HL (2009) Natural history, definitions, risk factors and burden of otitis media. The Medical Journal of Australia 191, S39-S43.

Labout JA, Duijts L, Lebon A, de Groot R, Hofman A, Jaddoe VV, Verbrugh HA, Hermans PW, Moll HA (2011) Risk factors for otitis media in children with special emphasis on the role of colonization with bacterial airway pathogens: the Generation $\mathrm{R}$ study. European Journal of Epidemiology 26, 61-66. doi:10.1007/s10654-010-9500-2

Morris PS, Leach AJ, Silberberg P, Mellon G, Wilson C, Hamilton E, Beissbarth J (2005) Otitis media in young Aboriginal children from remote communities in Northern and Central Australia: a cross-sectional survey. BMC Pediatrics 5, 27. doi:10.1186/1471-2431-5-27 
Paradise JL, Rockette HE, Colborn DK, Bernard BS, Smith CG, Kurs-Lasky M, Janosky JE (1997) Otitis media in 2253 Pittsburgh-area infants: prevalence and risk factors during the first two years of life. Pediatrics 99, 318-333. doi:10.1542/peds.99.3.318

Peduzzi P, Concato J, Kemper E, Holford TR, Feinstein AR (1996) A simulation study of the number of events per variable in logistic regression analysis. Journal of Clinical Epidemiology 49, 1373-1379. doi:10.1016/ S0895-4356(96)00236-3

Roberts JE, Rosenfeld RM, Zeisel SA (2004) Otitis media and speech and language: a meta-analysis of prospective studies. Pediatrics 113, e238-e248. doi:10.1542/peds.113.3.e238

Rosenkranz S, Abbott P, Reath J, Gunasekera H, Hu W (2012) Promoting diagnostic accuracy in general practitioner management of otitis media in children: findings from a multimodal, interactive workshop on tympanometry and pneumatic otoscopy. Quality in Primary Care 20, 275-285.
Schluter P, Carter S, Kokaua J (2007) Indices and perception of crowding in Pacific households domicile within Auckland, New Zealand: findings from the Pacific Islands Family Study. The New Zealand Medical Journal 120, U2393

Spurling GK, Askew DA, Schluter PJ, Simpson F, Hayman NE (2012) Conventionally accepted risk factors do not appear to explain higher rates of middle ear disease in remote Indigenous children: an ecological study. Australian and New Zealand Journal of Public Health 36, 491-492. doi:10.1111/j.1753-6405.2012.00921.x

Uhari M, Mäntysaari K, Niemelä M (1996) A meta-analytic review of the risk factors for acute otitis media. Clinical Infectious Diseases 22, 1079-1083. doi:10.1093/clinids/22.6.1079 